Ann. Geophysicae 14, 1019-1024 (1996) C EGS - Springer-Verlag 1996

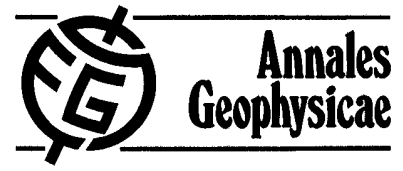

\title{
A generalization of the minimum variance analysis method
}

\author{
H. Kawano ${ }^{1}$, T. Higuchi ${ }^{2}$ \\ 1 Institute of Geophysics and Planetary Physics, University of California, Los Angeles, CA 90095-1567, USA \\ ${ }^{2}$ Institute of Statistical Mathematics, Tokyo 106, Japan
}

Received: 7 November 1995/Revised: 30 May 1996/Accepted: 14 June 1996

\begin{abstract}
In order to determine the normal direction of the magnetopause, the minimum variance analysis technique is frequently used: it is applied to the magnetic field data of a magnetopause crossing observed by a satellite, and provides the direction along which the magnetic field variation is minimum. In this study we propose a method to extend naturally the framework of the minimum variance analysis so that it includes all continuity conditions across a planar magnetopause, so that we could use all physical quantities observed by the spacecraft. We first extend it to the electric field: we discuss how to determine the normal direction and the speed of the magnetopause along the normal by using both the magnetic and electric field data. We next discuss the full extension to all continuity conditions across the magnetopause. Finally, we discuss how to extend our method further so that it can deal with the magnetopause which is accelerating. A discussion of application to observations will appear separately.
\end{abstract}

\section{Introduction}

In order to determine the normal direction of the magnetopause, Sonnerup and Cahill (1967) proposed a method called the minimum variance analysis method (denoted by MVA) applied to the magnetic field data, and since then the method has widely been used for that aim. A brief description of the MVA method being applied to the magnetic field data during the magnetopause crossings follows. The magnetopause is assumed to be planar and unchanging in time. Then, from Coulomb's Law for the magnetic field, $\operatorname{div} \mathbf{B}=0$, the magnetic field component normal to the magnetopause, $B_{n}$, is required to be constant across the magnetopause. In application to

Correspondence to: $\mathrm{H}$. Kawano actual observations, this condition would mean the minimum variance of the magnetic field along the normal. Then, MVA applies the eigenvalue analysis technique to the variance-covariance matrix of the magnetic field and determines such a direction.

The purpose of this work is to propose a new methodology to extend the framework of MVA in a natural manner so that it includes other boundary conditions across the planar magnetopause. As a first step, in Sect. 2 we describe a method which includes the boundary condition of both the magnetic and the electric field (the method is called EBMVA). The method also provides an estimate for the speed of the magnetopause along the normal. We also discuss the features of this method, including comparisons with another methods in literature. In Sect. 3 we generalize the methodology further, still in a natural manner, so that it could include all boundary conditions across a one-dimensional magnetopause (this fully generalized MVA is called GMVA). We also discuss related papers in literature. In Sect. 4 we present a generalization to the case of the accelerating magnetopause. Finally, Sect. 5 gives a summary.

\section{EBMVA}

First we assume the orientation of the magnetopause, or in other words, we assume a normal vector of the magnetopause, $\mathbf{n}$. (In this stage $\mathbf{n}$ is assumed, but later in this section we show how to determine the optimal n from observed data.) Then we determine the velocity of the magnetopause discontinuity, $U_{d}$, along the assumed n, from observed magnetic and electric field data. Here $U_{d}$ is actually the velocity relative to the spacecraft.

In order to express the components of vectors below, we define a coordinate system, $L M N$, which is based on $\mathbf{n}$ : its $N$ axis is parallel to $\mathbf{n}$, thus $L$ and $M$ are tangent to the magnetopause plane. Directions of the $L$ and $M$ axes on the magnetopause plane are arbitrarily defined. 
Under these assumptions, $U_{d}$ is analytically calculated by using the continuity condition of the tangential electric field in a frame moving with the magnetopause, as shown later. The electric field is obtained by direct observations or calculated by using the plasma velocity data, $\mathbf{V}$, and the MHD frozen-in condition, i.e.,

$\mathbf{E}=-\mathbf{V} \times \mathbf{B}$.

When the spacecraft observes the electric field $\mathbf{E}$, the electric field in the frame moving with the magnetopause, $\mathbf{E}^{\prime}$, satisfies

$\mathbf{E}^{\prime}=\mathbf{E}+U_{d} \mathbf{n} \times \mathbf{B}$,

or

$E_{L}^{\prime}=E_{L}-B_{M} U_{d}$

$E_{M}^{\prime}=E_{M}+B_{L} U_{d}$

$E_{N}^{\prime}=E_{N}$

in component expression.

Because $\partial \mathbf{B} / \partial t=0$ in the frame moving with the boundary, Faraday's equation is reduced to $\operatorname{rot} \mathbf{E}^{\prime}=0$, leading to the constancy of $\mathbf{E}_{t}^{\prime}$ across the boundary, where the subscript $t$ means the electric field components perpendicular to n, i.e., $L$ and $M$ components. Then, for actual data, the optimal $U_{d}$ should minimize the variation of $\mathbf{E}_{t}^{\prime}$ during the magnetopause crossing interval. If we define the variance of a dataset $\left[\mathbf{y}_{1}, \mathbf{y}_{2}, \ldots, \mathbf{y}_{K}\right]$ with each $\mathbf{y}_{k}(k=1, \ldots, K)$ having a dimension $J$ as

$\Lambda(\mathbf{y}) \equiv \frac{1}{K-1} \sum_{k=1}^{K}\left[\sum_{j=1}^{J}\left(y_{j, k}-\bar{y}_{j}\right)^{2}\right]$,

where $y_{j, k}$ is the $j$-th component of $\mathbf{y}_{k}$ and $\bar{y}_{j}$ is the mean of the $j$-th component, then the variation of $\mathbf{E}_{t}^{\prime}$ can be expressed by its variance, $\Lambda\left(\mathbf{E}_{t}^{\prime}\right)$ :

$\Lambda\left(\mathbf{E}_{t}^{\prime}\right)=\frac{1}{K-1}\left[\sum_{k=1}^{K}\left(E_{L, k}^{\prime}-\bar{E}_{L}^{\prime}\right)^{2}+\sum_{k=1}^{K}\left(E_{M, k}^{\prime}-\bar{E}_{M}^{\prime}\right)^{2}\right]$.

Minimization of $\Lambda\left(\mathbf{E}_{t}^{\prime}\right)$ is achieved by solving

$\frac{\partial \Lambda\left(\mathbf{E}_{t}^{\prime}\right)}{\partial U_{d}}=0$

and because $E_{L}^{\prime}$ and $E_{M}^{\prime}$ are linear functions of $U_{d}$, as shown in Eq. (2), the solution is given in an analytic form as follows:

$U_{d}=\frac{\sum_{k=1}^{K}\left(\mathbf{E}_{k} \times \mathbf{B}_{k}-\overline{\mathbf{E}} \times \overline{\mathbf{B}}\right)_{N}}{\sum_{k=1}^{K}\left(B_{L, k}-\bar{B}_{L}\right)^{2}+\sum_{k=1}^{K}\left(B_{M, k}-\bar{B}_{M}\right)^{2}}$,

where the numerator means the $N$ component of the vector.

In this way, the obtained $U_{d}$ provides the best condition for the continuity of the electric field tangential components, for the assumed $\mathbf{n}$. Next we find the optimal $\mathbf{n}$, i.e., the optimal direction of the magnetopause normal. In doing so, we also consider the continuity condition of the magnetic field normal components $B_{n}$, as described later.
Our plan to find the optimal $\mathbf{n}$ is to find a point over the entire unit sphere surface where $D_{n}$ becomes minimum, where $D_{n}$ is a certain function of $\mathbf{n}$ and should express to what extent $\mathbf{E}_{t}^{\prime}$ and $B_{n}$ are constant. Because the magnetic field and the electric field have different dimensions, there is no one-and-only way to define the functional form of $D_{n}$. In this study we adopt the definition as

$D_{n}=D_{n}(\mathbf{n}) \equiv \frac{\Lambda\left(B_{n}\right)}{\Lambda\left(B_{m}\right)} \cdot \frac{\Lambda\left(\mathbf{E}_{t}^{\prime}\right)}{\Lambda\left(E_{n}^{\prime}\right)}$,

whose meaning is described next.

In the first term of $D_{n}, B_{n}=B_{N}=\mathbf{B} \cdot \mathbf{n}$, and $B_{m}=\mathbf{B} \cdot \mathbf{m}$, where the unit vector $\mathbf{m}$ is defined so that it is perpendicular to $\mathbf{n}$ and so that the magnetic field variance in the two components perpendicular to $\mathbf{n}$ is smallest along $\mathbf{m}$. Under this definition, if $\mathbf{n}$ is set parallel to the minimum variance eigenvector of the magnetic field, $\mathbf{m}$ is then parallel to the intermediate variance eigenvector. Thus, the first term of the $D_{n}$ becomes minimum for the direction which MVA provides as the normal. We note here that the ratio of the minimum to intermediate variances is usually used to express the validity of MVA. The second term of the $D_{n}$ is related to the electric field and defined in a similar manner to the first term: the variance of the tangential components are divided by that of the normal component, to test the constancy of the tangential components in comparison with the normal component variation. The numerator, $\Lambda\left(\mathbf{E}_{t}^{\prime}\right)$, is the same as in Eq. (4), with $U_{d}$ determined by Eq. (6). In the denominator, $E_{n}^{\prime}=E_{N}^{\prime}=\mathbf{E}^{\prime} \cdot \mathbf{n}$. Because $\Lambda\left(B_{n}\right) / \Lambda\left(B_{m}\right)$ and $\Lambda\left(\mathbf{E}_{t}^{\prime}\right) / \Lambda\left(E_{n}^{\prime}\right)$ are both non-dimensional, the two terms are connected in $D_{n}$ by multiplication to express the conjugate condition minimizing $\Lambda\left(\mathbf{E}_{t}^{\prime}\right)$ and $\Lambda\left(B_{n}\right)$ at the same time. We regard this connection as a natural one, and note $D_{n}$ is usable as an extended version of the criterion which is usually used to express the validity of the results of MVA. That is, if $D_{n}$ is too large we may reject the obtained results.

The actual procedure to find $\mathbf{n}$ which minimizes $D_{n}(\mathbf{n})$ is achieved numerically: there are many publications which deal with the numerical minimization (e.g., Press et al., 1992) so one can refer to them for actual calculations. After the optimal $\mathbf{n}$ is determined in this way, the associated $U_{d}$ is already calculated, it is easy to calculate $\bar{B}_{n}$, and the deHoffman-Teller (HT) frame velocity $\mathbf{V}_{H T}$ can be calculated by

$\mathbf{V}_{H T}=\frac{\sum_{k=1}^{K} \mathbf{E}_{t, k}^{\prime} \times \mathbf{n}}{\sum_{k=1}^{K} B_{n, k}}$.

To summarize in order to obtain the optimal n, we minimize $D_{n}(\mathbf{n})$ over the unit sphere surface, or if we express $\mathbf{n}$ in the spherical coordinate system as $(\theta, \phi)$, we find the minimum point of $D_{n}(\theta, \phi)$ in the range of $0 \leq \theta \leq \pi$ and $0 \leq \phi \leq 2 \pi$. In order to calculate $D_{n}$ for a given set of $(\theta, \phi)$, we first use Eq. (6) to determine $U_{d}$, secondly use Eq. (1) to obtain $\mathbf{E}^{\prime}$, then calculate the variances of $\mathbf{B}$ and $\mathbf{E}^{\prime}$ appearing in Eq. (7) and obtain $D_{n}$.

Having described the EBMVA method, we note that Sonnerup et al. (1987) have presented a method which also uses the (motional inductive) electric field data and the magnetic field data to determine the normal direction and 
the magnetopause motion along the direction. When they assume the constant acceleration of the magnetopause they show how to specify the range of possible initial speed, but not the most probable value. On the other hand when they assume that the magnetopause is moving with a constant speed, it is usually difficult to specify the range of the possible speed or the most probable speed. On the other hand in our EBMVA method, the most probable value of the magnetopause normal speed is uniquely determined, even under the assumption of the constant motion of the magnetopause. (For the acceleration effect, see Sect. 4.) Another difference between the two methods is that their method explicitly uses the HT frame, which would narrow the range of its application: for example the HT frame does not exist for the transition layers of tangential discontinuities (TDs). On the other hand our method should be applicable to TDs also, because our method uses the most basic physical laws only.

We also note that Terasawa et al. (1996) present a method similar to EBMVA in this work. Here we briefly summarize their method. They start with $D / D t \equiv$ $\partial / \partial t+\mathbf{U}_{d} \cdot \nabla=0$, which holds true because the system is time-independent other than a constant motion $\mathbf{U}_{d}$. (Here $\mathbf{U}_{d} \equiv U_{d} \mathbf{n}$, and we note $\mathbf{V}_{C}$ in their paper corresponds to $\mathbf{U}_{d}$ here: here we use the notations in this study.) By using this relation, Faraday's law in the spacecraft frame is transformed, by using some vector algebra, as follows:

$\operatorname{rot} \mathbf{E}+\frac{\partial \mathbf{B}}{\partial t}=0$

$\operatorname{rot} \mathbf{E}-\left(\mathbf{U}_{d} \cdot \nabla\right) \mathbf{B}=0$

$\operatorname{rot}\left(\mathbf{E}+\mathbf{U}_{d} \times \mathbf{B}\right)-\mathbf{U}_{d} \operatorname{div} \mathbf{B}=0$

$\operatorname{rot} \mathbf{E}^{\prime}-\mathbf{U}_{d} \operatorname{div} \mathbf{B}=0$.

Then, assuming that the system is only a function of the $N$-position in the $L M N$ coordinates,

$$
\nabla=\mathbf{n} \frac{\partial}{\partial N}
$$

thus Faraday's law becomes

$\frac{\partial}{\partial N}\left(\mathbf{n} \times \mathbf{E}^{\prime}-U_{d} \mathbf{n} B_{N}\right)=0$.

Therefore:

$\left(\mathbf{n} \times \mathbf{E}^{\prime}-U_{d} \mathbf{n} B_{N}\right)=\mathbf{C}$,

where $\mathbf{C}$ is a vector constant. Using this relation, they minimize the mean square of $\left(\mathbf{n} \times \mathbf{E}^{\prime}-U_{d} \mathbf{n} B_{N}\right)-\mathbf{C}$ and determine $\mathbf{n}, U_{d}$, and $\mathbf{C}$.

The difference between EBMVA and Terasawa et al.'s (1996) method is apparent in the equation $\operatorname{rot} \mathbf{E}^{\prime}-$ $\mathbf{U}_{d} \operatorname{div} \mathbf{B}=0$. In EBMVA we only use $\operatorname{rot} \mathbf{E}^{\prime}=0$ as Faraday's law, which is correct because $\operatorname{div} \mathbf{B}=0$. In other words, $\operatorname{rot} \mathbf{E}^{\prime}=0$ is the essential part of Faraday's law in that it is covariant under the Lorentz transformation. However by keeping the $\mathbf{U}_{d} \operatorname{div} \mathbf{B}$ term in the equa- tion, they can couple the two conditions $\operatorname{rot} \mathbf{E}^{\prime}=0$ and $\operatorname{div} \mathbf{B}=0$, the former (latter) of which leads to the constancy of $\mathbf{E}_{t}^{\prime}\left(B_{N}\right)$. Their coupling method is, therefore, based on summation, while the coupling method of EBMVA is based on multiplication. As we stated earlier, there is no one-and-only way for the coupling, because the magnetic field and the electric field have different dimensions: with appropriate algorithms (including the determination of scaling parameters, which we will discuss next), multiplication-based methods and summationbased methods should both be valid. However from a practical viewpoint, summation-based methods would need more parameters to determine than the multiplication-based method presented in this work, especially when other boundary conditions such as the mass flux conservation are included. That is, when terms from different boundary conditions are added, scaling parameters are needed to equalize the dimensions of all terms. An example is $\mathbf{U}_{d}$ in the equation $\operatorname{rot} \mathbf{E}^{\prime}-\mathbf{U}_{d} \operatorname{div} \mathbf{B}=0$. Also, when an attempt is made to couple many boundary conditions, it is not necessarily clear how to define the scaling parameters, because the physical meaning of the scaling parameters is not always apparent: for example, note that any assumed $\mathbf{U}_{Q}$, having the dimension of velocity, satisfies $\operatorname{rot}\left(\mathbf{E}+\mathbf{U}_{d} \times \mathbf{B}\right)-\mathbf{U}_{Q} \operatorname{div} \mathbf{B}=0$. On the other hand, the multiplication-based coupling methodology in this study does not include such scaling parameters whose determination need additional consideration, and the methodology can be naturally extended to other boundary conditions such as the mass flux conservation, an extension we show in the next section.

Returning to the features of the EBMVA method, in actual observations it often happens that the sampling time is different between the magnetic field data and the electric field data (or the plasma velocity data which are used to calculate the motional induction electric field). Usually the sampling time is shorter for the magnetic field and thus the number of the magnetic field data points $\left(K_{B}\right)$ is larger than the number of the electric field data points $\left(K_{E}\right)$ obtained over the same time interval. In such a case, the first term of the right hand side of Eq. (7) can be calculated by using the $K_{B}$ magnetic field data, while the second term can be calculated by the $K_{E}$ sets of $(\mathbf{E}, \mathbf{B})$, where $\mathbf{B}$ is usually averaged to have the same sampling time as $\mathbf{E}$. However the problem here is that the functional form of Eq. (7) does not reflect the information of the quantity of data. In general, as the quantity of data increases, the standard error of any statistic calculated from the data decreases. So in Eq. (7), the standard error of $\Lambda\left(B_{n}\right) / \Lambda\left(B_{m}\right)$ for a fixed $\mathbf{n}$ decreases with increasing $K_{B}$. The same can be said for $\Lambda\left(\mathbf{E}_{t}^{\prime}\right) / \Lambda\left(E_{n}^{\prime}\right)$. Thus, the reliability, or weights, of the two terms should depend on the quantity of data, but Eq. (7) does not include such weights. Then, a possible definition of $D_{n}$ which includes the weights is as follows:

$D_{n} \equiv\left[\frac{\Lambda\left(B_{n}\right)}{\Lambda\left(B_{m}\right)}\right]^{K_{B} /\left(K_{B}+K_{E}\right)} \cdot\left[\frac{\Lambda\left(\mathbf{E}_{t}^{\prime}\right)}{\Lambda\left(E_{n}^{\prime}\right)}\right]^{K_{E} /\left(K_{B}+K_{E}\right)}$.

For example if $K_{B} \gg K_{E}$, only the first term remains in $D_{n}$ and the magnetic field data determine $\mathbf{n}$, which is the 
same as is obtained with MVA. Also note that instrumental conditions may affect the accuracy of observed physical quantities, as has been pointed out by Sonnerup et al. (1987). Some additional weights may therefore be applied depending on the instrumental conditions, in the same style as Eq. (9).

We note that in MVA and EBMVA we minimize variances $(\Lambda)$ in order to reach the optimum solution. However it is known that the method of minimizing the variance is too sensitive to outliers. For example, if one data point is distributed far from other data points, i.e., if it has a large deviation from the average of other data points, then that one point can distort considerably the result of minimization, because the deviation is squared in the variance which is to be minimized. If such outliers are removed by visual inspection, arbitrariness cannot be avoided in doing so. In order to overcome this problem, 68 percentile ranges can be used instead of variances, with the outliers kept in the dataset. For example, instead of using

$\Lambda\left(B_{n}\right)=\frac{1}{K-1} \sum_{k=1}^{K}\left(B_{n, k}-\bar{B}_{n}\right)^{2}$,

$B_{n}$ can be sorted in, say, ascending order, and the robust estimate of the variance defined by

$$
\begin{aligned}
\Delta\left(B_{n}\right) \equiv & \mid B_{n}^{*}(\langle K / 2+0.5+K r\rangle) \\
& -\left.B_{n}^{*}(\langle K / 2+0.5-K r\rangle)\right|^{2}
\end{aligned}
$$

can be used, where $B_{n}^{*}(i)$ refers to the $i$-th smallest data, $\langle y\rangle$ of a real number $y$ means the integer number nearest to $y, K$ is the total number of the data, $K r=0.34134 \cdot K$, and 0.34134 is a $1-\sigma$ range for a normalized Gaussian distribution. This 68 percentile range is unchanged if, for example, the largest data point becomes even larger, meaning that this quantity is robust for outliers.

\section{GMVA}

There are three other boundary conditions which are not included in EBMVA, i.e., the mass flux conservation:

$$
\begin{aligned}
\rho V_{n}^{\prime} & \equiv \mathbf{n} \cdot\left[\rho \mathbf{V}^{\prime}\right] \equiv \mathbf{n} \cdot\left[\rho\left(\mathbf{V}-U_{d} \mathbf{n}\right)\right] \\
& =\rho\left(V_{n}-U_{d}\right)=\text { const. },
\end{aligned}
$$

the momentum conservation:

$$
\begin{aligned}
\mathbf{S}^{\prime} & \equiv \rho\left(V_{n}-U_{d}\right)\left(\mathbf{V}-U_{d} \mathbf{n}\right)+\left(p+\frac{B^{2}}{2 \mu_{0}}\right) \mathbf{n}-\frac{1}{\mu_{0}} B_{n} \mathbf{B} \\
& =\text { const. }
\end{aligned}
$$

and the energy flux conservation:

$$
\begin{aligned}
T_{n}^{\prime} & \equiv \mathbf{n} \cdot \mathbf{T}^{\prime} \\
& \equiv \mathbf{n} \cdot\left[\left(\frac{1}{2} \rho \mathbf{V}^{\prime 2}+p+\varepsilon\right) \mathbf{V}^{\prime}+\frac{B^{2}}{\mu_{0}} \mathbf{V}^{\prime}-\frac{1}{\mu_{0}}\left(\mathbf{V}^{\prime} \cdot \mathbf{B}\right) \mathbf{B}+\mathbf{q}\right] \\
& =\left(\frac{1}{2} \rho \mathbf{V}^{\prime 2}+p+\varepsilon\right) V_{n}^{\prime}+\frac{B^{2}}{\mu_{0}} V_{n}^{\prime}-\frac{1}{\mu_{0}}\left(\mathbf{V}^{\prime} \cdot \mathbf{B}\right) B_{n}+q_{n} \\
& =\text { const. }
\end{aligned}
$$

where quantities observed at the spacecraft are shown without prime while quantities in the frame moving with the magnetopause are shown with prime, $\varepsilon$ refers to the internal energy density, and $\mathbf{q}$ refers to the heat flux. Here we note that Eq. (12) and Eq. (14) have a similar style: $\rho V_{n}^{\prime}$ is the normal component of $\rho \mathbf{V}^{\prime}$, and $T_{n}^{\prime}$ is the normal component of $\mathbf{T}^{\prime}$. Because this is similar to $B_{n}$ being the normal component of $\mathbf{B}$, we can include the contributions of $\rho V_{n}^{\prime}$ and $T_{n}^{\prime}$ into $D_{n}$ in the same manner as that used for $B_{n}$. That is, the constancy condition can be expressed in terms of the variance of the vector quantity $\left(\rho \mathbf{V}^{\prime}\right.$ or $\left.\mathbf{T}^{\prime}\right)$ along the normal direction, normalize (divide) it by the variance of the same vector data along a perpendicular direction, then multiply the normalized value to the right hand side of Eqs. (7) or (9). On the other hand, Eq. (13) means constancy of all three components of $\mathbf{S}^{\prime}$. In this case this method for normalization cannot be used, because there is no component which is to change during the magnetopause crossing. Therefore another way should be thought of to include this momentum conservation condition. An answer is to find an $\mathbf{n}_{S^{\prime} \max }$ which maximizes

$F_{S^{\prime}}(\mathbf{n}) \equiv \sum_{k=1}^{K}\left|\mathbf{S}_{k}^{\prime}-\overline{\mathbf{S}}^{\prime}\right|^{2}$

and to multiply $F_{S^{\prime}}(\mathbf{n}) / F_{S^{\prime}}\left(\mathbf{n}_{S^{\prime} \max }\right)$ to Eqs. (7) or (9). To summarize, if we write down the generalized version of Eq. (7) here, it is

$D_{n} \equiv \frac{\Lambda\left(B_{n}\right)}{\Lambda\left(B_{m_{B}}\right)} \cdot \frac{\Lambda\left(\mathbf{E}_{t}^{\prime}\right)}{\Lambda\left(E_{n}^{\prime}\right)} \cdot \frac{\Lambda\left(\rho V_{n}^{\prime}\right)}{\Lambda\left(\rho V_{m_{V}}^{\prime}\right)} \cdot \frac{F_{S^{\prime}}(\mathbf{n})}{F_{S^{\prime}}\left(\mathbf{n}_{S^{\prime} \max }\right)} \cdot \frac{\Lambda\left(T_{n}^{\prime}\right)}{\Lambda\left(T_{m_{T}}^{\prime}\right)}$,

where $\mathbf{m}_{B}$ is the same as $\mathbf{m}$ in Eqs. (7) and (9) (how to determine it is described in the previous section), and $\mathbf{m}_{V}$ and $\mathbf{m}_{T}$ unit vectors are obtained from $\rho \mathbf{V}^{\prime}$ and $\mathbf{T}^{\prime}$ by using the same procedure as is used to obtain $\mathbf{m}_{B}$ from $\mathbf{B}$.

As another feature of the GMVA method, Eq. (6) may not be used to determine $U_{d}$, because many terms of Eq. (16) include $U_{d}$. The reasonable solution to this problem is to solve the set of $\left(\mathbf{n}, U_{d}\right)$ numerically at once so that $D_{n}$ becomes minimum.

Finally we note that Sonnerup et al. (1987) applied MVA to the mass flux data of a certain magnetopause crossing event. The result was that the minimum variance was comparable to the intermediate variance, leading to poor determination of $\mathbf{n}$ from the mass flux data. That situation was also the case for $\mathbf{B}$ observed at the same time: $\mathbf{B}$ also had comparable values of the intermediate and minimum variances. Then, they did not use the mass flux data in their final method to determine $\mathbf{n}$, but they used the maximum variance eigenvector of $\mathbf{B}\left(\mathbf{i}_{B}\right)$ : they found that $\mathbf{i}_{B}$ is nearly tangential to the magnetopause, and constrained their method so that $\mathbf{n}$ must be perpendicular to $\mathbf{i}_{B}$ (see, e.g., page 12,150 of Sonnerup et al., 1987).

One may then ask what is the use of the $\mathbf{B}$ and $\rho \mathbf{V}^{\prime}$ data in GMVA (the first and third terms of Eq. 16) in a situation like this (comparable values of the two smallest eigenvalues for both the magnetic field and the mass flux), where the data do not provide a proper estimation of $\mathbf{n}$ by themselves. The answer is that, the inclusion of $\mathbf{B}$ and $\rho \mathbf{V}^{\prime}$ 
into GMVA is meaningful even in such a situation. To explain how it is so, let us first discuss $\mathbf{B}$ when $\mathbf{B}$ has the same values of the intermediate and minimum variances, for simplicity. In such a case, the first term of Eq. (16) (or 7), $\Lambda\left(B_{n}\right) / \Lambda\left(B_{m}\right)$, becomes unity for any assumed $\mathbf{n}$ if it is perpendicular to $\mathbf{i}_{B}$. On the other hand, if $\mathbf{n}$ is assumed to be parallel to $\mathbf{i}_{B}$, the term $\Lambda\left(B_{n}\right) / \Lambda\left(B_{m}\right)$ becomes larger than unity. Thus the unity is the minimum value $\Lambda\left(B_{n}\right) / \Lambda\left(B_{m}\right)$ can take, and the minimum value occurs not at a certain point but at a ring (great circle on the unit sphere surface) corresponding to n's satisfying $\mathbf{n} \cdot \mathbf{i}_{B}=0$. This means that the $\Lambda\left(B_{n}\right) / \Lambda\left(B_{m}\right)$ term in Eq. (16) tends to constrain the region of possible minimum of $D_{n}$ to the ring perpendicular to $\mathbf{i}_{B}$. Then other conditions such as that of $\mathbf{E}^{\prime}$ determine the solution as a point on or near the ring. This effect of $\mathbf{B}$ on the solution $\mathbf{n}$ is basically the same as is employed by Sonnerup et al. (1987) (using $\mathbf{i}_{B}$, as stated), although our method is more flexible in that the angle between $\mathbf{n}$ and $\mathbf{i}_{B}$ is not fixed to the exact $90^{\circ}$ (other boundary conditions' contribution in Eq. 16 can shift the minimal point of $D_{n}$ ).

Having discussed the magnetic field, we now discuss the mass flux. What we are looking at here is the situation where the mass flux data have comparable values of intermediate and the minimum variances. Then with the same logic as already discussed, the third term of Eq. (16), $\Lambda\left(\rho V_{n}^{\prime}\right) / \Lambda\left(\rho V_{m_{v}}^{\prime}\right)$, tends to constrain the solution $\mathbf{n}$ to a ring perpendicular to the maximum variance eigenvector of $\rho \mathbf{V}^{\prime}$. Thus, the inclusion of the mass flux term into Eq. (16) is meaningful. Both of the first and third terms act as constraints to the solution, in the situation discussed here, and their contributions are evenly and objectively evaluated in the form of multiplication in Eq. (16).

We also note that Paschmann et al. (1986) examined the tangential momentum conservation. Their conclusion was that the tangential momentum conservation was generally satisfied for $\mathbf{n}$ determined with the maximum variance analysis of the electric field. This information suggests that the momentum conservation condition can also lead to a proper estimation of $\mathbf{n}$, and thus it is favorable to our method.

\section{Magnetopause acceleration effect}

Sonnerup et al. (1987) included the acceleration effect of the magnetopause motion in their method. We note that it is also possible for our EBMVA method to include the acceleration effect, by assuming

$U_{d}=U_{0}+\alpha \tau$,

where $\tau$ is the time, offset to the start time of the observation, $U_{0}$ is the initial velocity, and $\alpha$ is the acceleration. Because this equation is linear with regard to $U_{0}$ and $\alpha$, Eq. (5) can again be solved analytically, and the solution is

$\left(\begin{array}{c}U_{0} \\ \alpha\end{array}\right)=\mathbf{M}^{-1} \xi$ where $\mathbf{M}$ is a matrix whose elements are

$$
\begin{aligned}
M_{11}= & \sum_{k=1}^{K}\left(B_{L, k}^{2}+B_{M, k}^{2}\right)-\frac{1}{N}\left\{\left(\sum_{k=1}^{K} B_{L, k}\right)^{2}\right. \\
& \left.+\left(\sum_{k=1}^{K} B_{M, k}\right)^{2}\right\} \\
M_{12}= & \sum_{k=1}^{K}\left(B_{L, k}^{2}+B_{M, k}^{2}\right) \tau_{k}-\frac{1}{N}\left(\sum_{k=1}^{K} B_{L, k} \sum_{k=1}^{K} B_{L, k} \tau_{k}\right. \\
& \left.+\sum_{k=1}^{K} B_{M, k} \sum_{k=1}^{K} B_{M, k} \tau_{k}\right) \\
M_{21}= & M_{12} \\
M_{22}= & \sum_{k=1}^{K}\left(B_{L, k}^{2}+B_{M, k}^{2}\right) \tau_{k}^{2}-\frac{1}{N}\left\{\left(\sum_{k=1}^{K} B_{L, k} \tau_{k}\right)^{2}\right. \\
& \left.+\left(\sum_{k=1}^{K} B_{M, k} \tau_{k}\right)^{2}\right\},
\end{aligned}
$$

and $\xi$ is a vector whose components are

$$
\begin{aligned}
\xi_{1}= & \sum_{k=1}^{K}\left(E_{L, k} B_{M, k}-E_{M, k} B_{L, k}\right) \\
& -\frac{1}{N}\left(\sum_{k=1}^{K} E_{L, k} \sum_{k=1}^{K} B_{M, k}-\sum_{k=1}^{K} E_{M, k} \sum_{k=1}^{K} B_{L, k}\right) \\
\xi_{2}= & \sum_{k=1}^{K}\left(E_{L, k} B_{M, k}-E_{M, k} B_{L, k}\right) \tau_{k} \\
& -\frac{1}{N}\left(\sum_{k=1}^{K} E_{L, k} \sum_{k=1}^{K} B_{M, k} \tau_{k}-\sum_{k=1}^{K} E_{M, k} \sum_{k=1}^{K} B_{L, k} \tau_{k}\right) .
\end{aligned}
$$

Other procedures are entirely the same as in the previous section. For the GMVA method, this equation may not be used but the set of $\left(\mathbf{n}, U_{0}, \alpha\right)$ has to be solved numerically at once so that $D_{n}$ becomes minimum, because $U_{0}$ and $\alpha$ are then included in many terms of Eq. (16).

\section{Summary}

We have proposed a methodology to generalize naturally the concept of MVA to physical quantities other than the magnetic field. We believe the physical meaning of the methodology is clear and simple enough to lend reliability to the methodology by itself. We expect that this methodology is able to draw meaningful information from the magnetopause crossing data for which MVA does not work good. Tests of the methodology by simulation studies, application to actual observations, and comparisons with other methodologies, will be presented in a future publication.

Acknowledgements. We thank T. Terasawa for useful discussions. This work has partly been supported by the Japanese Society for the Promotion of Science. 
Topical Editor K.-H. Glaßmeier thanks M. Dunlop and another referee for their help in evaluating this paper.

\section{References}

Paschmann, G., I. Papamastorakis, W. Baumjohann, N. Sckopke, C. W. Carlson, B. U. Ö. Sonnerup, and H. Lühr, The magnetopause for large magnetic shear: AMPTE/IRM observations, J. Geophys. Res., 91, 11,099-11,115, 1986.

Press, W. H., S. A. Teukolsky, W. T. Vetterling, and B. P. Flannery, Numerical recipes in fortran: the art of scientific computing, second edition, Cambridge University Press, 1992.
Sonnerup, B. U. Ö., and L. J. Cahill Jr., Magnetopause structure and altitude from Explorer-12 observations, J. Geophys. Res., 72, 171-183, 1967.

Sonnerup, B. U. Ö., I. Papamastorakis, G. Paschmann, and H. Lühr, Magnetopause properties from AMPTE/IRM observations of the convection electric field: method development, J. Geophys. Res., 92, 12,137-12,159, 1987.

Terasawa, T., H. Kawano, I. Shinohara, T. Mukai, Y. Saito, M. Hoshino, A. Nishida, S. Machida, T. Nagai, T. Yamamoto, and S. Kokubun, On the determination of a moving MHD structure: minimization of the residue of integrated Faraday's equation, J. Geomagn. Geoelectr., in press, 1996. 